\title{
THE NEUROMUSCULAR JUNCTION DISORDERS
}

Marguerite Hill

J Neurol Neurosurg Psychiatry 2003;74(Suppl II):ii32-ii37

$\mathrm{N}$ euromuscular junction (NMJ) disorders result from destruction, malfunction or absence of one or more key proteins involved in neuromuscular transmission, illustrated diagrammatically in fig 1 . The most common pathology is antibody mediated damage or down regulation of ion channels or receptors, resulting in myasthenia gravis (MG), Lambert-Eaton myasthenic syndrome (LEMS), and acquired neuromyotonia (Isaac's syndrome). Not surprisingly these three conditions share many common features (table 1). A second important group of disorders are the congenital myasthenic syndromes caused by mutations in NMJ proteins. Detailed discussion of these rare conditions is beyond the scope of this short review but interested readers are referred to a recent review by Engel and Ohno. ${ }^{1}$

\section{MYASTHENIA GRAVIS}

\section{Pathophysiology}

In anti-AChR antibody positive MG, autoantibodies target the acetylcholine (ACh) receptor $(\mathrm{AChR})$ resulting in receptor blockade, down regulation, and complement mediated destruction, thus reducing the number of receptors available to interact with the ACh released from the presynaptic nerve terminal. Complement activation attracts activated macrophages, which cause significant damage to the synaptic folds and loss of voltage gated sodium channels, which in turn increases the threshold required to initiate a muscle action potential. The consequence of the combined loss of AChRs and sodium channels is that the safety factor for neuromuscular transmission is reduced, and transmission at many endplates fails.

Although it has long been suspected that "seronegative" MG is also antibody mediated, it was only recently that one of the target proteins, muscle specific kinase (MuSK), was identified. ${ }^{2}$ During ontogeny this protein appears to play a key role in aggregation of AChRs at the motor end plate; its role during adult life is not yet clear.

\section{Epidemiology}

The prevalence of MG in the UK is estimated to be 15 per 100000 with an incidence of 1.1/100 000 population per year. ${ }^{3}$ To put this in context, MG is about seven times less common than multiple sclerosis, and as common as motor neurone disease in the over 60 age group. In women the incidence is bimodal with one peak between the ages of 16-35 years and a second over the age of 65, while in men MG is predominantly a disease of later life. Anti-AChR antibody positive MG is rare before puberty in white Europeans. Rarely MG is caused by drugs, in particular the antirheumatic drug, D-penicillamine. Drug induced disease generally, but not invariably, remits on stopping the offending agent.

\section{Making the diagnosis \\ Clinical features}

In theory, the fatiguable weakness characteristic of MG should be easy to recognise. In practice there is often a significant time lag between symptom onset and diagnosis. There are a number of reasons for this; to non-neurologists $M G$ is a rare disease and more common diagnoses such as a stroke, motor neurone disease or hysteria are likely to be entertained first. The fluctuating nature of the condition can also create diagnostic confusion; the double vision that was such a problem when watching television the night before will have often resolved when reviewed by a doctor the following morning. Eliciting fatigue is not part of the "routine" neurological examination, so you have to think of MG before you can find it. Furthermore, it should be remembered that not all patients that fatigue have MG and not all myasthenics clearly fatigue.

Correspondence to: Dr Marguerite Hill, Institute of Clinical Neurosciences, Frenchay Hospital, Bristol BS 16 1LE, UK;

marguerite.hill@bristol.ac.uk

\section{Investigations}

Anti-ACh receptor antibodies

A clearly positive anti-ACh antibody titre in a patient with a clinical picture of fatiguable weakness is enough to confirm the diagnosis of MG. False positive antibody results are very rare. Equivocal levels can occur in other autoimmune conditions and are more difficult to interpret, and 


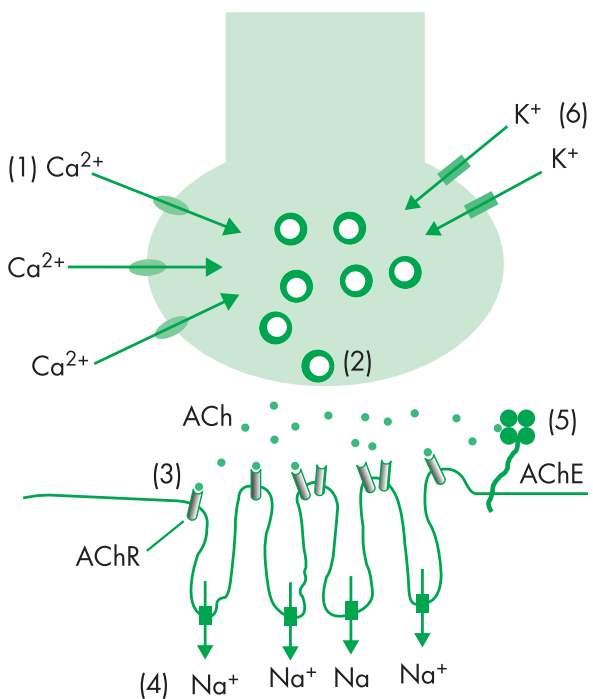

Figure 1 Diagrammatic representation of neuromuscular transmission. (1) Action potential arriving at nerve terminal triggers opening of voltage gated calcium channels (VGCCs) and entry of calcium. (2) Rise in intracellular calcium triggers release of packets of acetylcholine (ACh). (3) Interaction of ACh with ACh receptors (AChR) depolarises post-synaptic membrane. (4) Voltage gated sodium channels (VGSCs) open, triggering muscle action potential. (5) ACh esterase (AChE) breaks ACh into acetyl and choline, which are taken up by the nerve terminal to be reformed into $A C h$. (6) Opening of voltage gated potassium channels (VGKCs) repolarises nerve terminal.

neurophysiology should be performed to confirm the diagnosis. The real problem comes with antibody negative patients. It is estimated that about $50 \%$ of patients with purely ocular MG and $15 \%$ of those with generalised symptoms do not have antibodies against the AChR. Of these, just under half will be seropositive for MuSK antibodies; in the remainder one must rely on clinical symptoms, signs, and the results of detailed neurophysiology.
The Tensilon test

The Tensilon (edrophonium) test has been largely superseded by the anti-AChR antibody assay and detailed neurophysiology in the diagnosis of MG, though it still has a place, particularly when the diagnosis needs to be confirmed with some degree of urgency. Although one of the few "magic tricks" in medicine, the test itself poses a number of potential pitfalls for the unwary. Firstly it carries a small but not insignificant risk of respiratory arrest and cardiac arrhythmias, and should be used with caution in anyone with a history of cardiac arrhythmias or chronic respiratory disease. The test should always be performed where there are full resuscitation facilities available and patients pre-dosed with atropine. Secondly, a positive test is not $100 \%$ specific for MG and can be seen in a range of conditions including motor neurone disease and botulism. Conversely a negative test does not exclude the diagnosis. Many people advocate performing the test in a double blind fashion; in practice this is difficult to achieve, as the muscarinic side effects of edrophonium are generally clear to both patient and observer, and because its duration of action may be prolonged. You could try performing the test single blind with saline or atropine as the first "test" dose or simply accept that there may be a placebo response.

\section{Neurophysiological studies}

Repetitive nerve stimulation involves supramaximal stimulation at $3 \mathrm{~Hz}$ of a peripheral nerve, often the ulnar nerve, and recording the compound motor action potential (CMAP) from the relevant muscle. A decrement of more than $10 \%$ over five responses is considered consistent with a diagnosis of MG. Unfortunately the test is unreliable if the limb is cold or if the patient has taken ACh esterase (AChE) inhibitors, and it is often normal in ocular myasthenia. Patients may find supramaximal stimulation quite uncomfortable. A more sensitive test is single fibre electromyography (SFEMG), where recordings are made from two muscle fibres in a single motor unit. Increased variability in the interval between paired action potentials, termed 'jitter', or occasional blocking of one of the potentials is considered evidence of a defect in neuromuscular transmission. However, increased jitter can be

\begin{tabular}{|c|c|c|c|c|}
\hline & $\begin{array}{l}\text { "Seropositive" myasthenia } \\
\text { gravis }\end{array}$ & $\begin{array}{l}\text { "Seronegative" myasthenia } \\
\text { gravis }\end{array}$ & $\begin{array}{l}\text { Lambert Eaton myasthenic } \\
\text { syndrome }\end{array}$ & Acquired neuromyotonia \\
\hline Antigenic target & Acetylcholine receptor & $\begin{array}{l}\text { Muscle specific kinase + } \\
\text { unknown antigen(s) }\end{array}$ & Voltage gated calcium channel & Voltage gated potassium channel \\
\hline Associations & $\begin{array}{l}\text { Idiopathic: other autoimmune } \\
\text { conditions } \\
\text { Paraneoplastic: thymoma }\end{array}$ & & $\begin{array}{l}\text { Idiopathic: other autoimmune } \\
\text { conditions } \\
\text { Paraneoplastic: small cell lung } \\
\text { cancer }\end{array}$ & $\begin{array}{l}\text { Idiopathic: other autoimmune } \\
\text { conditions } \\
\text { Paraneoplastic: thymoma, small } \\
\text { cell lung cancer }\end{array}$ \\
\hline $\begin{array}{l}\text { Effect of removal of } \\
\text { underlying tumour }\end{array}$ & $\begin{array}{l}\text { No effect on neurological } \\
\text { symptoms }\end{array}$ & & $\begin{array}{l}\text { Resolution of neurological } \\
\text { symptoms }\end{array}$ & $\begin{array}{l}\text { No effect on neurological } \\
\text { symptoms }\end{array}$ \\
\hline Neurophysiology & $\begin{array}{l}\text { (1) } 10 \% \text { decrement on } \\
\text { repetitive nerve stimulation } \\
\text { (2) Increased jitter and } \\
\text { blocking SFEMG }\end{array}$ & $\begin{array}{l}\text { (1) } 10 \% \text { decrement on } \\
\text { repetitive nerve stimulation } \\
\text { (2) Increased jitter and } \\
\text { blocking on SFEMG }\end{array}$ & $\begin{array}{l}\text { (1) } 50 \% \text { increase in CMAP after } \\
\text { max voluntary contraction } \\
\text { (2) Increased jitter on SFEMG }\end{array}$ & $\begin{array}{l}\text { (1) Spontaneous neuromyotonic } \\
\text { discharges } \\
\text { (2) Fasciculation and fibrillation } \\
\text { potentials }\end{array}$ \\
\hline Response to steroids & $\begin{array}{l}\text { Good, but may show initial } \\
\text { deterioration }\end{array}$ & Good & Good & Variable \\
\hline $\begin{array}{l}\text { Response to plasma } \\
\text { exchange }\end{array}$ & Good & Good & Good & Good \\
\hline Response to IVlg & Good & Variable & Good & Variable \\
\hline
\end{tabular}

CMAP, compound motor action potential; IVlg, intravenous immunoglobulin; SFEMG, single fibre electromyography. 


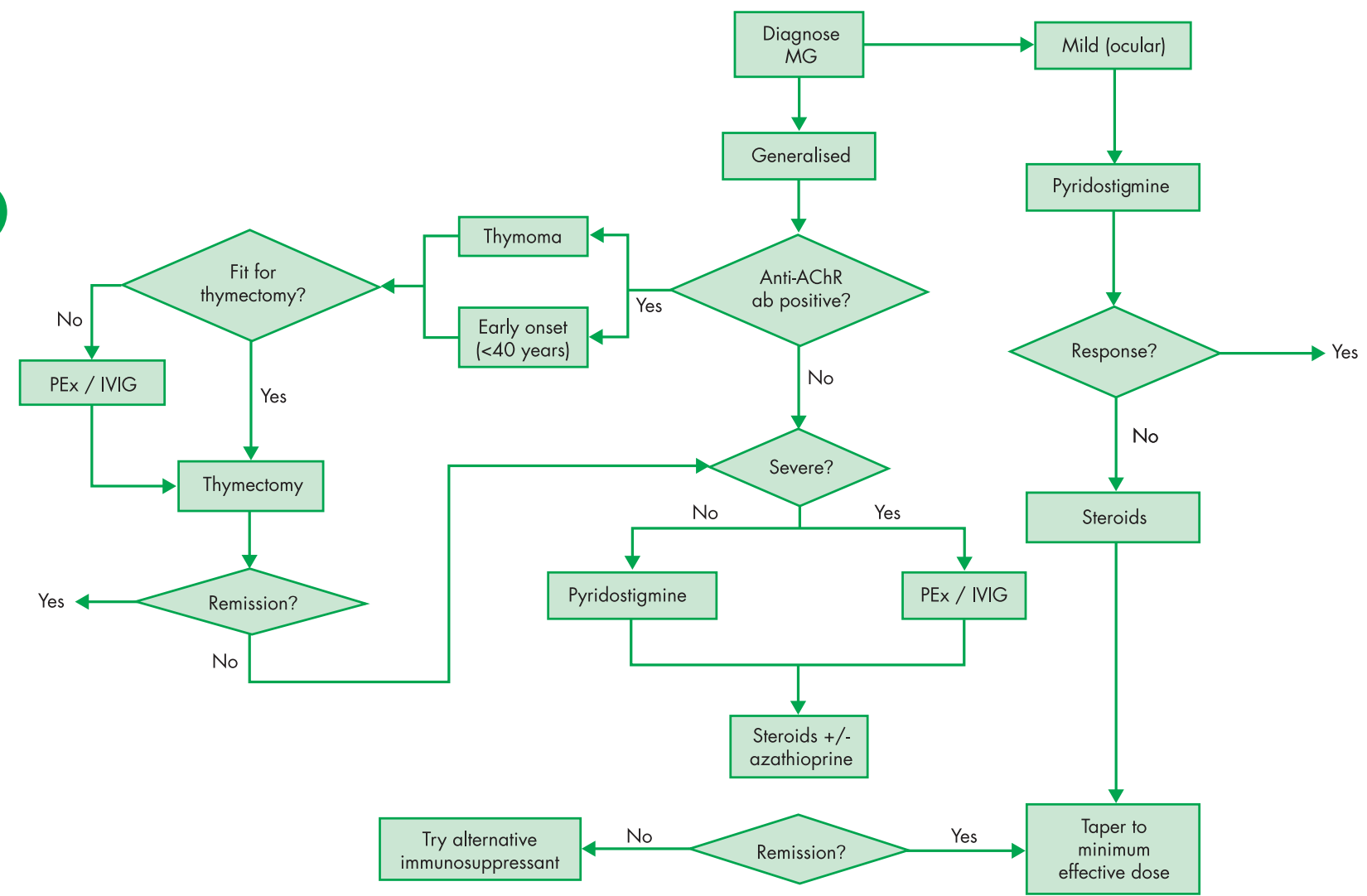

Figure 2 Treatment algorithm for myasthenia gravis (MG). AChR, acetylcholine receptor; IVIG, intravenous immunoglobulin; PEx, plasma exchange.

found in conditions other than MG and so needs to be carefully interpreted. Furthermore it is a test that demands a high degree of expertise, which may not be available locally, and again patients find it quite uncomfortable particularly when the orbicularis oculi is tested.

\section{Management}

In the era of evidence based medicine, the management of the neuromuscular disorders has lagged far behind many other conditions. This is not surprising for diseases such as LEMS and neuromyotonia whose rarity makes any sort of randomised controlled trial a challenge, to say the least. However, it is disappointing that we have little hard evidence on which to base our management of MG, and there are probably as many different treatment regimens for myasthenia as there are neurologists. Hopefully over the next few years we will be able to move from anecdotal to evidence based treatment for this condition. Figure 2 shows a treatment algorithm for MG.

\section{Managing mild myasthenia}

Patients with mild myasthenia-that is, those with ocular symptoms and no bulbar weakness-can be managed on an outpatient basis, though at symptom onset it is impossible to be sure that the disease is not going to progress. The first line of treatment is the oral AChE inhibitor pyridostigmine, starting with $30 \mathrm{mg}$ three times daily and gradually increasing according to response to $60-90 \mathrm{mg}$ four times daily. Gastrointestinal side effects are common and can be extremely debilitating, although antimuscarinics such propantheline (15-30 mg three times daily) may provide some relief. Cholinergic crises caused by excessive doses of pyridostigmine are less common than they used to be because immunosuppressant drugs tend to be introduced earlier in the disease process, but they are still a risk and the patient should be counselled against taking too many tablets.

Many patients with mild disease respond well to pyridostigmine, and will not require more intensive treatment. However, a significant proportion of ocular myasthenics notice little benefit. In these cases there needs to be a careful discussion with the patient about the pros and cons of trying steroids or managing with practical aids such as eyelid props. If they wish to start steroids, and you are satisfied that they have purely ocular myasthenia, then steroids can be started slowly as an outpatient. I generally start with $5 \mathrm{mg}$ alternate days, increasing by $5 \mathrm{mg}$ per week up to a maximum dose of between 20-30 mg alternate days. Rarely it may be necessary to go up to $1 \mathrm{mg} / \mathrm{kg}$ to achieve remission. Patients should always be warned to report immediately should they develop speech or swallowing difficulties.

\section{Managing moderate to severe myasthenia}

Any myasthenic with worsening generalised disease needs to be admitted to hospital, whether they are newly diagnosed or established patients in the throes of a relapse. Starting or increasing the dose of pyridostigmine may be of benefit, though rapidly escalating doses are a clear sign that immunosuppressant treatment needs to be initiated. In those patients unable to swallow, neostigmine (1-2.5 mg subcutaneously) may be helpful, although the incidence of muscarinic side effects with this drug is high and the production of excess secretions may be life threatening. In general if a patient is bad enough to require neostigmine, they are bad enough to require monitoring in intensive care.

Although steroids have been the mainstay of treatment for MG for three decades, dosing regimens are based on 
experience rather than evidence. The main concern is that initiating steroids may induce an initial deterioration in symptoms before any benefit is realised, although the "safe" speed of introduction has not been established. It is generally reasonable to start at $10 \mathrm{mg} /$ day and increase by $10 \mathrm{mg}$ every second day to a maintenance dose of $1.5 \mathrm{mg} / \mathrm{kg}$, or $100 \mathrm{mg}$, whichever is the lower. This should be maintained until symptoms resolve, after which they can be slowly reduced. Whether you aim to stabilise the patient on a daily or alternate day regimen is a matter of personal preference. There have never been any randomised trials demonstrating the benefit of alternate day steroids, and patients may find it confusing or notice an increase in symptoms on the "non-steroid" day. However, many experienced practitioners feel that the incidence of side effects, in particular steroid myopathy, is reduced on an alternate day regimen. The speed of reduction should be gauged by the severity of the patient's MG, their prior success or otherwise in reducing steroids, and their tolerance of side effects. However, I would not reduce more rapidly than $5 \mathrm{mg} / \mathrm{month}$ down to $20 \mathrm{mg}$ alternate days, then by $2.5 \mathrm{mg} / \mathrm{month}$ to $10 \mathrm{mg}$, and then by a maximum of $1 \mathrm{mg} / \mathrm{month}$. Many people relapse when their steroid dose falls below $10 \mathrm{mg}$ alternate days and therefore serious consideration needs to be given to the addition of another immunosuppressant drug when the steroids are started.

The low frequency of spontaneous remission in MG coupled with the high incidence of steroid induced side effects with prolonged use means that the use of other immunosuppressant drugs as "steroid sparing agents" is now widespread. The most popular is azathioprine $(2.5 \mathrm{mg} / \mathrm{kg} / \mathrm{day})$ — the subject of one of the few double blinded randomised controlled trials in MG. ${ }^{4}$ As the benefit of azathioprine is not realised for about 12-18 months, it should be started at the same time as steroids in any patient with moderate to severe disease. Unfortunately a significant number of people experience fevers, chills, nausea, and vomiting on azathioprine, and like most immunosuppressants hepatic function and white blood count needs to be monitored regularly. After azathioprine, cyclosporin $(2-5 \mathrm{mg} / \mathrm{kg} /$ day adjusted according to blood values) is the only immunosuppressant that has been subject to a randomised controlled trial. ${ }^{5}$ Although this study showed that the drug was beneficial, $35 \%$ of participants had to withdraw because of side effects, in particular nephrotoxicity. Anecdotally, methotrexate (7.5-25 mg weekly) also appears to be effective and patients appear to respond quicker than they do to azathioprine. There are reports of benefit using some of the newer immunosuppressants such as mycophenolate and tacrolimus; however, in the absence of any objective evidence of their efficacy, or indeed superiority, over more established and cheaper drugs, it is difficult to justify their use until other treatments have failed.

\section{Managing a myasthenic crisis}

Myasthenic crises can occur in undiagnosed myasthenics, where it is important that the condition is considered early and appropriate treatment instituted, or in established patients precipitated by infection, treatment with drugs that interfere with neuromuscular transmission such as the aminoglycoside antibiotics, or inadequate immunosuppression. Immediate concerns in a crisis are deteriorating respiratory function and dysphagia. The former is best assessed by regular measurements of the forced vital capacity, though it can be difficult to get accurate readings in the elderly and those with pronounced facial weakness. Other useful markers of respiratory failure are an increasing respiratory rate, having to take a breath after two or three words, and being unable to count to 10 slowly out loud on a single breath. Patients whose vital capacity is dropping rapidly or who can only achieve $15 \mathrm{ml} / \mathrm{kg}$ should be admitted to intensive care. The integrity of a patient's swallow can be quickly assessed by watching them drink $10 \mathrm{ml}$ of water to see if they cough, choke or have a "bubbly" voice afterwards. Neostigmine before meals may also be helpful but if there is any doubt about the safety of their swallow, they should be placed nil by mouth until formally assessed. Aspiration pneumonia is clearly an undesirable outcome in someone with already compromised respiratory function.

Once the safety of the patient is established, steroid treatment should be started or increased. If they are already requiring ventilatory support the maximum dose of steroids should be instituted immediately. However, it can take several weeks before the benefit of steroids are realised, in which case plasma exchange or infusion of intravenous immunoglobulin (IVIg) should be considered to induce a more rapid, albeit short lived, remission. A randomised direct comparison of the two showed them to be equally effective. ${ }^{6}$ IVIg is generally better tolerated but the benefit is seen earlier with plasma exchange. In my experience more patients respond to plasma exchange, particularly if they are "seronegative", although many hospitals have limited access to plasma exchange services, particularly "on call", and so IVIg is generally the more practical option. However, if the patient has not responded seven days after initiation of IVIg treatment, it is reasonable to try plasma exchange if possible.

The role of thymectomy

The association between anti-AChR antibody positive myasthenia and thymic abnormalities is well recognised. Approximately $10 \%$ of patients have a thymoma, while early onset disease (before the age of 40) is associated with thymic hyperplasia. A computed tomographic (CT) thorax scan is therefore mandatory in all anti-AChR antibody positive patients. A thymoma should be removed because of the risk of metastatic spread within the thoracic cavity, but this will not result in disease remission. Thymectomy in the absence of a thymoma is more controversial. A recent analysis of published data on the subject concluded that the operation was probably beneficial in patients with severe disease. ${ }^{7}$ However, as this conclusion was based on analysis of non-randomised retrospective studies of a heterogeneous population of patients, it is difficult to draw too many conclusions, except that a well designed trial is urgently needed. At the moment it is probably fair to say that thymectomy is likely to be followed by remission over three years in about $25 \%$ of patients and some improvement in a further $50 \%$ in anti-AChR antibody positive patients with early disease onset (before the age of 40), although this advice may change in light of new evidence over the next decade.

\section{LAMBERT EATON MYASTHENIC SYNDROME (LEMS) Pathophysiology}

In LEMS the autoantibody target is the P/Q type voltage gated calcium channel expressed on the presynaptic motor nerve terminals, autonomic nerve terminals, and cerebellar Purkinje cells. Loss of this ion channel leads to a reduction in calcium entry into the nerve terminal, thus insufficient ACh is released to elevate the end plate potential above that required for sodium channel activation. Repeated impulses increase the amount of calcium entering the nerve terminal and eventually 
sufficient ACh is released to trigger a muscle action potential. Thus in LEMS, sustained effort increases muscle strengthpost-tetanic potentiation.

\section{Epidemiology}

Although there is no accurate epidemiological data for LEMS,

it is probably more than 10-fold less common than MG. In $60 \%$ of cases it is associated with an underlying small cell lung cancer (SCLC) and a prospective study detected LEMS in 3\% of these patients. ${ }^{8}$ Neurological symptoms may precede radiological evidence of tumour by some years, so it is probably prudent to scan high risk patients (that is, smokers) every year. Non-cancer associated LEMS may occur in childhood or in adult life at any age, and not infrequently coexists with other autoimmune conditions.

\section{Making the diagnosis}

\section{Clinical features}

The first symptom noticed by the patient is difficultly walking, generally because of proximal leg weakness but also occasionally because of ataxia. Arms may also be affected but ocular, bulbar, and respiratory symptoms are unusual, although well recognised. Patients often report that they are worse in the heat. On direct questioning patients often admit to a variety of autonomic symptoms such as dry mouth, constipation, impotence, and bladder urgency. The classic textbook findings on examination are a demonstrable increase in muscle power muscle and a reappearance of absent reflexes with sustained effort, though this can be difficult to demonstrate in practice.

\section{Investigations}

Anti-VGCC (voltage gated calcium channel) antibodies can be detected in the patient's serum in over $90 \%$ of cases, and like anti-AChR antibodies, are extremely specific for LEMS. Typical findings on neurophysiology are a notably reduced CMAP, with a dramatic increase after maximum voluntary contraction.

\section{Management}

The first line of treatment for LEMS is the drug 3,4 diaminopyridine (3,4-DAP), which blocks presynaptic potassium channels, thereby increasing the opening time of the available VGCC. A reasonable starting dose is $10 \mathrm{mg}$ four times daily, which can be further increased to $20 \mathrm{mg}$ four times daily if required. Patients should be warned that they may experience perioral and peripheral paraesthesia. Doses above $100 \mathrm{mg}$ a day are not recommended because of the risk of seizures (the drug is contraindicated in epilepsy), and there are reports that the drug can precipitate asthma attacks in susceptible people. Some patients also find that pyridostigmine provides moderate benefit, though generally it is less pronounced than that seen in MG.

In LEMS associated with SCLC, appropriate treatment for the tumour should be instituted. Unlike thymoma and MG, the induction of oncological remission is reflected by improvement in the neurological symptoms. Non-cancer patients who remain symptomatic despite maximal treatment with 3,4-DAP should be started on steroids and azathioprine, which retrospective studies have shown to be of benefit. As with MG, plasma exchange or IVIg will induce rapid but temporary remission in severely affected patients. ${ }^{9}$

\section{NEUROMYOTONIA \\ Pathophysiology}

In acquired neuromyotonia, autoantibodies cause down regulation of voltage gated potassium channels (VGKCs) expressed on the peripheral nerve terminal. This reduction in VGKCs

\section{Abbreviations}

ACh: acetylcholine

AChE: acetylcholine esterase

AChR: acetylcholine receptor

CMAP: compound motor action potential

IVIg: intravenous immunoglobulin

LEMS: Lambert Eaton myasthenic syndrome

MG: myasthenia gravis

MUSK: muscle specific kinase

NMJ: neuromuscular junction

SFEMG: single fibre electromyography

VKCC: voltage gated calcium channel

VGKC: voltage gated potassium channel

VGSC: voltage gated sodium channel

SCLC: small cell lung cancer

prolongs depolarisation of the nerve terminal, so increasing the amount of ACh released from the nerve terminal resulting in nerve hyperexcitability.

\section{Clinical features}

Acquired autoimmune neuromyotonia (Isaac's syndrome) is rare. It can occur at any age but the median age at onset is between 30-40 years. Most cases are idiopathic, though about $15 \%$ are associated with thymoma, and a small proportion with SCLC. The neuronal hyperexcitability results in skeletal muscle overactivity, which manifests as muscle twitching or myokymia (which is often confused with fasciculation), cramps, muscle stiffness, and in chronic cases, muscle hypertrophy. Increased sweating, presumably caused by continuous muscle activity, is also a common complaint.

\section{Investigations}

In common with MG and LEMS, diagnosis of neuromyotonia is based on positive titres of anti-VGKC antibodies and characteristic findings on neurophysiology. However, the immunoprecipitation assay used by most laboratories only detects antibodies in about $50 \%$ of cases; more sensitive tests can improve the yield to $87 \%$ but are not widely available. ${ }^{10}$ The classical EMG findings are of spontaneous doublet, triplet or multiple discharges from motor nerves. Fasciculation and fibrillation potentials are also found and some patients may have evidence of a mild peripheral neuropathy.

\section{Management}

Many patients gain significant symptomatic relief from drugs that down regulate voltage gated sodium channels such as the anticonvulsants carbamazepine, phenytoin, and lamotrigine. Plasma exchange can provide short term relief; the response to IVIg is generally less striking. Resistant cases may benefit from long term immunosuppression with prednisolone and azathioprine, though not all patients respond to this. It goes without saying that any underlying tumour should be treated appropriately, though like MG this is unlikely to result in neurological remission.

\section{CONCLUSIONS}

Autoimmune disorders of the NMJ, although uncommon, are a fascinating group of diseases, both clinically and scientifically. Although the pathophysiology of these conditions is now well understood, management is still largely based on anecdote and personal preference, and there is a clear need for well designed randomised trials to clarify the optimal treatment for these diseases. 


\section{ACKNOWLEDGEMENTS}

I am grateful to Professor John Newsom-Davis for critical reading of this article.

\section{REFERENCES}

1 Engel AG, Ohno K. Congenital myasthenic syndromes. Adv Neurol 2002;88:203-15

A valuable review of this fast moving field.

2 Hoch W, McConville J, Helms S, et al. Auto-antibodies to the receptor tyrosine kinase MUSK in patients with myasthenia gravis without acetylcholine receptor antibodies. Nat Med 2001;7:365-8.

- A significant advance in our understanding of "seronegative" MG.

3 Robertson NP, Deans J, Compston DA. Myasthenia gravis: a population based epidemiological study in Cambridgeshire, England. J Neurol Neurosurg Psychiatry 1998;65:492-6.

4 Palace J, Newsom-Davis J, Lecky B. A randomized double-blind trial of prednisolone alone or with azathioprine in myasthenia gravis. Myasthenia gravis study group. Neurology 1998;50:1778-83.
One of the few randomised controlled trials in MG which has significantly influenced current management.

5 Tindall RS, Phillips JT, Rollins JA, et al. A clinical therapeutic trial of cyclosporine in myasthenia gravis. Ann N Y Acad Sci 1993;681:539-51.

6 Gaidos P, Chevret S, Clair B et al. Clinical trial of plasma exchange and high-dose intravenous immunoglobulin in myasthenia gravis. Myasthenia gravis clinical study group. Ann Neurol 1997;41:789-96

7 Gronseth GS, Barohn RJ. Practice parameter: thymectomy for autoimmune myasthenia gravis (an evidence-based review): report of the quality standards subcommittee of the American Academy of Neurology. Neurology 2000;55:7-15.

8 Elrington GM, Murray NM, Spiro SG, et al. Neurological paraneoplastic syndromes in patients with small cell lung cancer. A prospective survey of 150 patients. J Neurol Neurosurg Psychiatry 1991;54:764-7.

9 Newsom-Davis J. A treatment algorithm for Lambert-Eaton myasthenic syndrome. Ann N Y Acad Sci 1998;841:817-22.

- A practical guide to management of this uncommon condition.

10 Hart IK. Autoimmune neuromyotonia. In: Rose MR, Griggs RC, eds. Channelopathies of the nervous system. Oxford: Butterworth-Heinemann 2001:214-26.

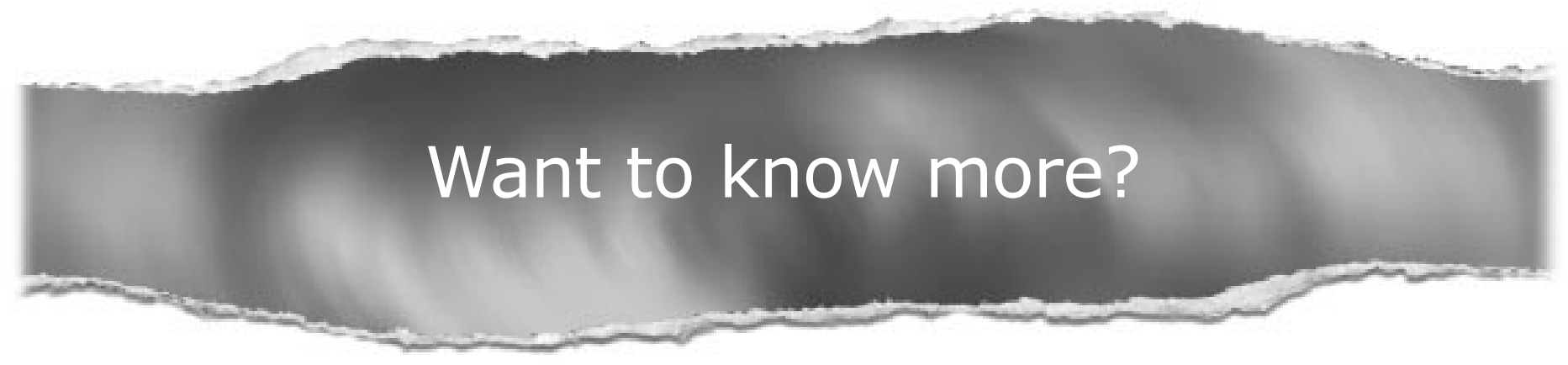

\section{Data supplements}

Limited space in printed journals means that interesting data and other material are often edited out of articles; however, limitless cyberspace means that we can include this information online.

Look out for additional tables, references, illustrations.

www.jnnp.com 